\title{
T-Wave Alternans Identification in Direct Fetal Electrocardiography
}

\author{
Ilaria Marcantoni, Marica Vagni, Angela Agostinelli, Agnese Sbrollini, Micaela Morettini, \\ Luca Burattini, Francesco Di Nardo, Sandro Fioretti, Laura Burattini \\ Università Politecnica delle Marche, Ancona, Italy
}

\begin{abstract}
Very little is known about the incidence and etiology of fetal T-wave alternans (TWA), an electrophysiologic phenomenon potentially associated to fetal suboptimal outcomes. Thus, availability of automatic methods for quantification of TWA from digital electrocardiograms (ECG) is desirable, since TWA occurrence might indicate the need of taking actions before or during delivery. The heart-rate adaptive match filter (HRAMF) is a wellestablished method to identify TWA in adult ECG. Aim of the present study was to investigate the possibility of using HRAMF to identify and quantify TWA also in direct fetal ECG (DFECG) recordings. To this aim, HRAMF was applied to 5 min-long DFECG acquired during delivery ("Abdominal and Direct Fetal Electrocardiogram Database” by Physionet) of five healthy fetuses. Significant levels of TWA were measured in all DFECG. Specifically, on average, TWA was quite high in amplitude $(9 \pm 2 \mu V)$ and variable in time, as indicated by values of standard deviation $(6 \pm 2 \mu V)$ and maximum $(28 \pm 10 \mu \mathrm{V})$ of TWA amplitude. Eventually, a positive correlation $(\rho=0.68)$ was observed between maximum TWA and fetal heart rate, even though the limited number of recordings makes this result preliminary. In conclusion, HRAMF proved to be a suitable tool to automatically identify TWA from DFECG.
\end{abstract}

\section{Introduction}

Cardiac repolarization anomalies, visible as $\mathrm{T}$-wave abnormalities in the electrocardiogram (ECG), are indicators of severe (sometimes lethal) cardiovascular diseases and cardiac instabilities. Most of them render the heart susceptible to lethal ventricular tachyarrhythmias, at any age, also before birth [1]. T-wave abnormalities manifest in a lot of different forms, among which T-wave alternans (TWA) has received particular attention because strongly related to sudden cardiac death [2]. TWA is a beat-to-beat alternation of the T-wave morphology, which may involve T-wave amplitude, shape and/or polarity. Although macroscopic TWA is a marker for a more severe cardiac instability than microscopic TWA, the latter is also considered clinically significant [2], is much more commonly found, but requires specifically designed automatic techniques to be identified. Reliable automatic TWA detection, quantification, interpretation and classification in adult ECG is quite challenging, since strongly dependent on heart rate (HR), HR variability (HRV), and quality of ECG signal [3-5]. Therefore, TWA investigation in fetuses is considerably more complex since fetal ECG (FECG) is characterized by a fast HR, is low-amplitude and, most of all, is affected by several interferences, artifacts and noises.

TWA incidence and characteristics in fetal populations are still to be determined. Fetal TWA has been occasionally observed in studies on magnetocardiographic recordings $[1,2,6]$ that underlie the potential importance of fetal TWA assessment as a result of the high incidence of TWA in fetuses with cardiac arrhythmias and suboptimal outcomes [1]. Moreover, also in fetuses TWA is often observed in association with QT prolongation; thus, an accurate long QT syndrome diagnosis linked to TWA identification might permit an efficient treatment in utero $[1,6]$. Therefore, fetal TWA is potentially the cause of some nowadays unexplained fetal demises and its detection might become the key to solve many currently unexplained severe problems, some of which leading fetus to death. Due to the importance of the topic, not supported until now by a suitable amount of data, the present study has the principal purpose of providing a proper tool for detecting and measuring TWA on direct FECG (DFECG) recordings. Thus, this study aims to propose the heart-rate adaptive match filter (HRAMF; a well-established method to identify TWA in adult ECG recordings [7]) for fetal TWA identification, and to test it in clinical DFECG.

\section{Data and Methods}

\subsection{Clinical data}

Clinical data consisted in the DFECG recordings available at the "Abdominal and Direct Fetal Electrocardiogram Database" [8] from Physionet [9]. Each recording (sampling frequency: $1 \mathrm{kHz}$ ) is $5 \mathrm{~min}$ - 
long and was directly acquired by placing a spiral electrode on the fetus scalp during the labor of five different women, all between $38^{\text {th }}$ and $41^{\text {th }}$ week of gestation. All DFECG recordings were acquired in the Department of Obstetrics at the Medical University of Silesia on healthy fetuses.

\subsection{Automatic T-wave alternans identification from direct fetal electrocardiograms}

DFECG windows were recursively extracted and preprocessed in order to establish their suitability for automatic TWA identification. When considered suitable, a DFECG window was submitted to HRAMF for TWA detection and quantification in terms of its amplitude (TWAA; $\mu$ V; Fig. 1).

Windowing. For each 5 min DFECG, 35 s DFECG windows were recursively (every second) extracted for a total of 265 extracted moving windows.

Preprocessing. DFECG windows were initially lowpass filtered (bidirectional $6^{\text {th }}$-order Butterworth filter; cut-off frequency: $35 \mathrm{~Hz}$ ) in order to remove high frequency and line noise. Then, fetal $\mathrm{R}$ peaks were detected using a version of the Pan-Tompkins' algorithm, specifically adjusted for fetal applications [10]. Baseline was subtracted from DFECG after having been estimated as a $3^{\text {rd }}$-order spline interpolation of fiducial points located $50 \mathrm{~ms}$ before R peaks. Then, DFECG windows were resized in order to include only the 32 central beats. Detected $\mathrm{R}$ peaks in each resized window were used to estimate mean RR interval (MRR), strictly linked to HR (MRR reciprocal); HRV (RR-interval standard deviation); and TWA frequency (TWAF, by definition equal to the reciprocal of twice MRR). Median beat was computed and correlated against each beat in the window. If correlation was lower than 0.70 , the beat was replaced with the median one since considered an ectopic beat or an artifact.

Suitability test. Once preprocessed, the resized DFECG window was tested to evaluate its suitability for TWA identification. Specifically, if $\mathrm{HRV} \leq 0.1 \cdot \mathrm{MRR}$ and there were at most two replaced beats the window was considered suitable, otherwise it was rejected.

Heart-rate adaptive match filter. HRAMF [7] uses TWAF to adaptively design a band-pass filter with a narrow $0.12 \mathrm{~Hz}$-wide passing band whose central frequency corresponds to TWAF. The filter is implemented as a $6^{\text {th }}$-order bidirectional Butterworth band-pass filter. When fed with a DFECG window, HRAMF provides a pseudosinusoidal signal that, if really pertaining to TWA, has its maxima and minima in correspondence of ST segments (or T waves). In this case, TWAA is computed as twice the pseudosinusoid amplitude, otherwise TWAA is set to zero.

\subsection{Statistics}

For each 5 min DFECG, the number of windows (NW) from which TWA was identified, together with mean TWAF (MTWAF), mean TWAA (MTWAA), maximum (MAXTWAA) and standard deviation (SDTWAA) of TWAA, all evaluated over NW, were computed. Trend of TWA with HR was evaluated by computing the Pearson's correlation coefficient ( $\rho$ ) between MTWAA and MAXTWAA against HR. Statistical significance was set at 0.05 .

\section{Results}

A summary of TWA features, as measured in $5 \mathrm{~min}$ DFECG recordings of the 5 fetuses, are reported in Table 1. HR, and thus TWAF were quite homogeneous among fetuses $(129 \pm 3 \mathrm{bpm}$ and $1.07 \pm 0.02 \mathrm{~Hz}$, respectively). Instead, intra-window HRV and noise level were quite subject-dependent, so that rejection rate was quite

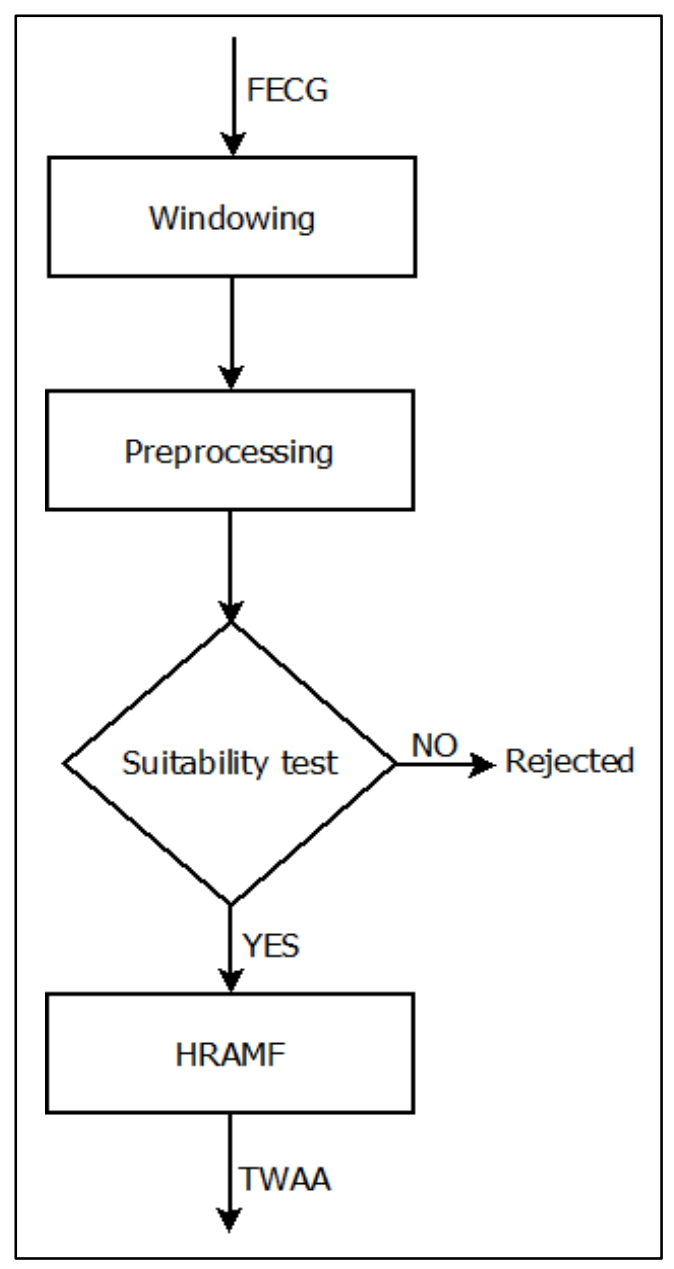

Fig. 1. Block diagram of the procedure for automatic TWA identification from DFECG. 
variable ( $8 \%$ to $80 \%)$. MTWAA, MAXTWAA and SDTWAA were all variable among fetuses, ranging from $6 \mu \mathrm{V}$ to $12 \mu \mathrm{V}$, from $17 \mu \mathrm{V}$ to $41 \mu \mathrm{V}$, and from $5 \mu \mathrm{V}$ to 9 $\mu \mathrm{V}$, respectively. Within each subject, MTWAA and SDTWAA were always of the same order of magnitude, being SDTWAA, on average, $72 \pm 6 \%$ of MTWAA. This result indicates a great TWAA variability along each DFECG recording (Fig. 2).

Correlation between MTWAA and HR was negligible $(\rho=0.12)$, whereas correlation between MAXTWAA and HR was quite high $(\rho=0.68$; Fig. 3). Statistical significance was never reached because of the limited number of subjects.

\section{Discussion}

The principal purpose of this study was identifying and quantifying TWA on DFECG by means of HRAMF, a method previously proposed and tested for adult TWA identification. The choice of using this specific method among the several present in literature $[3,4]$ is due to the HRAMF robustness deriving from its theoretical approach. Indeed, HRAMF is based on a narrowbandwidth filtering procedure which permits to keep only the frequency component pertaining to TWA (i.e. TWAF) while filtering out all other components related to interferences, artifacts and noises affecting DFECG [7].

Since used for the first time in fetal applications, HRAMF was applied here only to DFECG, obtained by positioning an electrode on the fetal scalp during delivery. With respect to indirect FECG, DFECG is less affected by noise and interferences, thus more suitable for TWA identification. However, its invasiveness limits its clinical use. Future studies will investigate the possibility of using

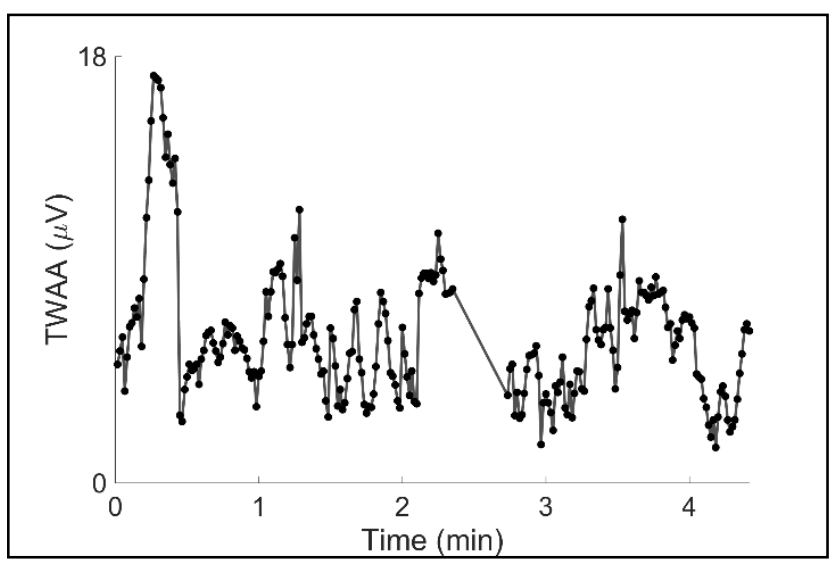

Fig 2. TWAA variation in time (DFECG number 1).

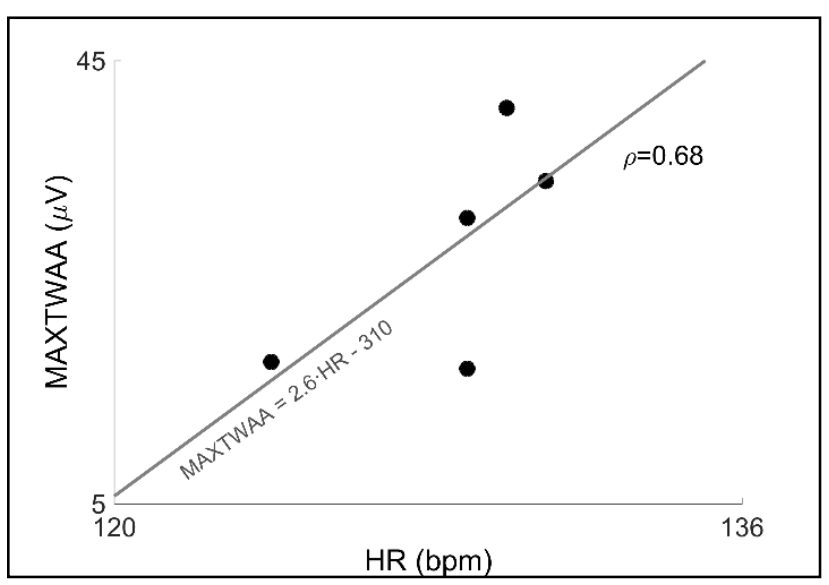

Fig. 3. Regression line between MAXTWAA and HR.

Table 1. TWA features over the population. Where suitable, values are reported as mean \pm standard deviation

\begin{tabular}{ccccccccc}
\hline DFECG & $\begin{array}{c}\text { HR } \\
(\mathrm{bpm})\end{array}$ & $\begin{array}{c}\text { MRR } \\
(\mathrm{ms})\end{array}$ & $\begin{array}{c}\text { HRV } \\
(\mathrm{ms})\end{array}$ & $\begin{array}{c}\text { TWAF } \\
(\mathrm{Hz})\end{array}$ & NW & $\begin{array}{c}\text { MTWAA } \\
(\mu \mathrm{V})\end{array}$ & $\begin{array}{c}\text { MAXTWAA } \\
(\mu \mathrm{V})\end{array}$ & $\begin{array}{c}\text { SDTWAA } \\
(\mu \mathrm{V})\end{array}$ \\
\hline 1 & 129 & 466 & $\begin{array}{c}14 \\
(3 \%)\end{array}$ & 1.07 & $\begin{array}{c}243 \\
(92 \%)\end{array}$ & 6 & 17 & 5 \\
2 & 129 & 466 & $\begin{array}{c}23 \\
(5 \%)\end{array}$ & 1.08 & $\begin{array}{c}177 \\
(67 \%)\end{array}$ & 8 & 31 & 5 \\
3 & 124 & 483 & $\begin{array}{c}10 \\
(2 \%)\end{array}$ & 1.04 & $\begin{array}{c}52 \\
(20 \%)\end{array}$ & 9 & 18 & 6 \\
4 & 131 & 459 & $\begin{array}{c}15 \\
(3 \%)\end{array}$ & 1.09 & $\begin{array}{c}237 \\
(89 \%) \\
203\end{array}$ & 9 & 34 & 7 \\
5 & 130 & 460 & $\begin{array}{c}23 \\
(5 \%)\end{array}$ & 1.09 & $\begin{array}{c}203 \%) \\
(77 \%)\end{array}$ & 12 & 41 & 9 \\
\hline \multirow{2}{*}{ Total } & $129 \pm 3$ & $467 \pm 9$ & $\begin{array}{c}17 \pm 6 \\
(4 \% \pm 1 \%)\end{array}$ & $1.07 \pm 0.02$ & $\begin{array}{c}182 \pm 78 \\
(69 \% \pm 29 \%)\end{array}$ & $9 \pm 2$ & $28 \pm 10$ & $6 \pm 2$ \\
\hline
\end{tabular}

HR: heart rate; MRR: mean RR interval; HRV: heart-rate variability; TWAF: T-wave alternans frequency; NW: number of windows in which T-wave alternans was identified; MTWAA: mean T-wave alternans amplitude; MAXTWAA: maximum of T-wave alternans amplitude; SDTWAA: standard deviation of T-wave alternans amplitude. 
HRAMF to identify TWA from indirect FECG, and will have to deal with high levels of noise, interferences and artifacts affecting it [11] and with the fact that TWAA is usually lead-dependent [12] (typically, indirect FECG recordings are multi-lead [13]).

The main finding of this paper is that fetuses show TWA, even though in healthy conditions, when usually TWA in adults is considered an indicator of cardiac risk [5]. This result is in agreement with one of the few papers on fetal TWA previously reported [2]. MAXTWAA was of the order of tens of $\mu \mathrm{V}$, that is similar to what observed in healthy adults in resting conditions [14]. However, DFECG amplitude is much lower than adult ECG and fetal data acquisition occurred during delivery which is a fetal stressful status not comparable to resting. Consequently, comparable TWAA values measured in fetuses vs adults might indicate that the phenomenon is much more significant in fetuses than adults, so that such measures should be considered high when referring to fetuses. High fetal TWA levels might also be related to the fact that fetuses show a much higher HR, and TWAA is known to be directly associated to it [5]. Here, such association was observed also for DFECG $(\rho=0.68)$ but this result has to be considered preliminary given the small number of cases in which it was evaluated.

Another preliminary but important finding of this paper is that TWA was strongly variable in time, and future studies will evaluate if this occurs independently or in relation to decelerations and bradycardia, i.e. when fetal complications are known to occur [15].

\section{Conclusion}

HRAMF proved to be a suitable tool to automatically identify TWA from DFECG. During delivery, fetuses typically show TWA, which is high in amplitude and variable in time.

\section{References}

[1] Zhao H, Strasburger JF, Cuneo BF, Wakai RT. Fetal cardiac repolarization abnormalities. Am J Cardiol 2006;98:491-6.

[2] Yu S, Van Veen BD, Wakai RT. Detection of T-wave alternans in fetal magnetocardiography using the generalized likelihood ratio test. IEEE Trans Biomed Eng 2013;60:2393-400.

[3] Burattini L, Bini S, Burattini R. Comparative analysis of methods for automatic detection and quantification of microvolt T-wave alternans. Med Eng Phys 2009;31:12908.

[4] Burattini L, Bini S, Burattini R. Automatic microvolt Twave alternans identification in relation to ECG interferences surviving preprocessing. Med Eng Phys
2011;33:17-30.

[5] Bloomfield DM, Hohnloser SH, Cohen RJ. Interpretation and Classification of Microvolt T Wave Alternans Tests. J Cardiovasc Electrophysiol 2002;13:502-12.

[6] Cuneo BF, Strasburger JF, Yu S, Horigome H, Hosono T, Kandori A, Wakai RT. In Utero Diagnosis of Long QT Syndrome by Magnetocardiography. Circulation 2013;128:2183-91.

[7] Burattini L, Zareba W, Burattini R. Automatic detection of microvolt T-wave alternans in Holter recordings: Effect of baseline wandering. Biomed Signal Process Control 2006;1:162-8.

[8] Jezewski J, Matonia A, Kupka T, Roj D, Czabanski R. Determination of the fetal heart rate from abdominal signals: evaluation of beat-to-beat accuracy in relation to the direct fetal electrocardiogram. Biomed Tech 2012;57:383-94.

[9] Goldberger AL, Amaral LA, Glass L, Hausdorff JM, Ivanov PC, Mark RG, Mietus JE, Moody GB, Peng CK, Stanley HE. PhysioBank, PhysioToolkit, and PhysioNet: components of a new research resource for complex physiologic signals. Circulation 2000;101:e215-20.

[10] Agostinelli A, Marcantoni I, Moretti E, Sbrollini A, Fioretti $S$, Di Nardo F, Burattini L. Noninvasive fetal electrocardiography part I: Pan-Tompkins' algorithm adaptation to fetal R-peak identification. Open Biomed Eng J 2017;11:17-24.

[11] Agostinelli A, Sbrollini A, Burattini L, Fioretti S, Di Nardo F, Burattini L. Noninvasive fetal electrocardiography part II: Segmented-beat modulation method for signal denoising. Open Biomed Eng J 2017;11:25-35.

[12] Burattini L, Man S, Burattini R, Swenne CA. Comparison of standard versus orthogonal ECG leads for T-wave alternans identification. Ann Noninvasive Electrocardiol 2012;17:130-40.

[13] Agostinelli A, Grillo M, Biagini A, Giuliani C, Burattini L, Fioretti S, Di Nardo F, Giannubilo SR, Ciavattini A, Burattini L. Noninvasive fetal electrocardiography: an overview of the signal electrophysiological meaning, recording procedures, and processing techniques. Ann Noninvasive Electrocardiol 2015;20:303-13.

[14] Burattini L, Zareba W, Burattini R. Identification of gender-related normality regions for T-wave alternans. Ann Noninvasive Electrocardiol 2010;15:328-36.

[15] Agostinelli A, Palmieri F, Biagini A, Sbrollini A, Burattini L, Di Nardo F, Fioretti S, Burattini L. Relationship between deceleration areas in the second stage of labor and neonatal acidemia. Comput Cardiol 2016;43:897-900.

Address for correspondence.

Laura Burattini.

Università Politecnica delle Marche,

Department of Information Engineering,

Via Brecce Bianche,

60131 Ancona, Italy.

E-mail address.1.burattini@univpm.it. 